\title{
Patient outcomes, patient experiences and process indicators associated with the routine use of patient-reported outcome measures (PROMs) in cancer care: a systematic review
}

\author{
Caitlin Graupner ${ }^{1,2}$ (D) $\cdot$ Merel L. Kimman ${ }^{3} \cdot$ Suzanne Mul ${ }^{1} \cdot$ Annerika H. M. Slok $^{4} \cdot$ Danny Claessens $^{4} \cdot$ Jos Kleijnen $^{5,6}$. \\ Carmen D. Dirksen ${ }^{3}$. Stéphanie O. Breukink ${ }^{1,2}$
}

Received: 12 May 2020 / Accepted: 19 August 2020 / Published online: 2 September 2020

(C) The Author(s) 2020

\begin{abstract}
Purpose In current cancer care, there is a growing debate about the value of using patient-reported outcome measures (PROMs) in daily clinical follow-up. A systematic review of the literature was conducted to assess the evidence of the effectiveness of the routine use of PROMs in daily cancer care in terms of patient outcomes, patient experiences and process indicators and to identify the effect of giving feedback about PROM findings to patients and/or health care professionals (HCPs).

Methods A systematic search was performed. Studies were eligible for inclusion when they (1) used a PROM as an intervention, with or without feedback to patients and/or HCPs, compared with not using a PROM, and (2) used a PROM as an intervention with feedback to patients and/or HCPs, compared with using a PROM without giving feedback to patients and/or HCPs.

Results After screening of 8341 references, 22 original studies met the inclusion criteria. Most studies found a positive effect on survival, symptoms, HRQoL and patient satisfaction. In general, using feedback to patient and/or HCPs about the PROM results led to better symptom control, HRQoL, patient satisfaction and patient-doctor communication. The majority of included studies had insufficient power to detect significant differences in the outcomes assessed.

Conclusion This review shows that predominantly positive findings were found in the use of a PROM in daily cancer care. Additionally, more positive effects were seen when feedback is provided to patient and/or health care professionals, and it is thus highly recommended that this is always done.
\end{abstract}

Keywords Patient-reported outcome measures $\cdot$ PROM $\cdot$ Cancer $\cdot$ Patient-reported outcomes

Caitlin Graupner

caitlin.graupner@mumc.nl

1 Department of Surgery, Maastricht University Medical Center, P. Debyelaan 25, 6229 HX Maastricht, The Netherlands

2 School for Oncology and Developmental Biology (GROW), Maastricht University, Maastricht, The Netherlands

3 Department of Clinical Epidemiology and Medical Technology Assessment, Maastricht University Medical Center and Care and Public Health Research Institute (CAPHRI), Maastricht, The Netherlands

4 Department of Family Medicine, Care and Public Health Research Institute (CAPHRI), Maastricht University, Maastricht, The Netherlands

5 Kleijnen Systematic Reviews Ltd, York, UK

6 Care and Public Health Research Institute (CAPHRI), Maastricht University, Maastricht, The Netherlands

\section{Abbreviations \\ PRO Patient-reported outcome \\ PROM Patient-reported outcome measure \\ HRQoL Health-related quality of life \\ HCP Health care professional \\ ICHOM International Consortium of Health \\ Outcomes Measurement}

\section{Introduction}

With improved cancer treatment modalities, the number of cancer survivors is rising [1]. For years, clinicians only focussed on traditional oncological outcomes, such as mortality and morbidity, in order to evaluate treatment effectiveness [2]. While survival and detection of recurrence are still the main pillars of cancer care follow-up, monitoring patient-centred outcomes, such as health-related quality of life (HRQoL), 
independence or fatigue, is now an accepted component of follow-up care [3]. This shift is also reflected in several oncological outcome sets of International Consortium of Health Outcomes Measurement (ICHOM) [4-6].

It is well known that cancer patients may have a high symptom burden which is caused either by the disease itself or their treatment modalities [7-9]. Underreporting of these symptoms by patients and underassessment by caregivers could result in under recognition and under treatment of these symptoms in daily oncological practice $[10,11]$. By using patientreported outcome measures (PROMs), the patient's perception of the impact of their condition or treatment on their quality of life can be evaluated and, if necessary, acted upon [12]. PROMs can focus on perceived needs, symptoms, response to treatment, undesirable side effects, effect on function or other aspects of the treatment that matter to patients and their families. Besides, PROMs can be used in shared decision-making throughout the entire process from diagnosis to follow-up [10, 13, 14]. Aggregated PROM data may also serve other purposes, such as quality improvement processes, clinical research and internal and external benchmarking [15].

In current cancer care, there is a growing debate about the additional value of using PROMs in daily clinical follow-up. Several oncological studies indicated that the systematic use of PROMs is associated with improved patient-physician communication [16], higher patient satisfaction [12] and improvement of patient symptom control [17]. A review by Kotronoulas et al. (2014) focused on PROM use in cancer care and investigated the effect of PROMs with and without individualized management plan [18]. The outcomes of this review focused on patient outcomes, process of care and health service outcomes. Their search was performed in 2013, and they included 26 studies in their review. They concluded that using PROMs increased the discussion of patient outcomes during consultations and that PROMs were associated with an improved symptom control and patient satisfaction. A recent systematic review by Ishaque et al. (2019) investigated the effectiveness of PROMs as an intervention to support the representation of patient values and preferences in clinical encounters in oncology $(n=12)$ and non-oncology settings $(n=10)$ [19]. They described overall positive findings in favour of the PROM intervention, especially when PROM results were shared with clinicians.

Opponents declare that the evidence of the additional value of PROMs is limited. Completing PROMs may be stressful and time-consuming for patients. A known barrier of using PROMs in daily care is the amount of extra administrative efforts experienced by health care professionals (HCPs) [20-22]. A properly functioning IT system could solve some of these extra efforts, yet this is often lacking [23].

The aim of this systematic review is to provide an up-todate evidence synthesis of the effectiveness of routine use of PROMs in daily cancer care, in terms of patient outcomes and experiences. The secondary objective is to identify the effect of giving feedback about PROM findings to patients and/or health care professionals compared with PROM use without any feedback.

\section{Methods}

\section{Search}

A systematic search was performed in September 2018 in MEDLINE, EMBASE, Cochrane Library Database, PubMed and CINAHL. Studies published within the last 20 years (1998-2018) were included. An update of the search was performed in December 2019. The search terms were chosen in such a way that any description that could resemble or relate to the use of PROMs within oncology would be discovered by the search (Appendix Table 5). Additional articles were identified by examining the reference lists of reviewed articles. No language restrictions were applied. Studies included in systematic reviews and meta-analyses were checked for eligibility.

\section{Study selection criteria}

Preferred Reporting Items for Systematic Reviews and MetaAnalyses (PRISMA) guidelines were followed throughout the review process [24]. Study selection consisted of a two-phase process performed by three researchers (CG, LM and SM). First, titles and abstracts were screened, and potentially eligible articles were retrieved independently by two researchers (CG and LM). Second, full-text reports were read by two authors (CG and SM), independently, to determine eligibility of the studies. Furthermore, the reference lists of eligible studies were checked for any missing studies. In case of disagreement in one of the two phases, a third reviewer (MK) was consulted until agreement was reached.

For the first aim, studies were eligible for inclusion when they used a PROM as intervention, with or without feedback to patients or health care professionals, compared with not using a PROM. For the second aim, studies were eligible when they used a PROM as intervention with feedback to patients or health care professionals, compared with a control group in which PROMs were used without giving feedback to patients or health care professionals about the results. All types of cancer patients were included, and no specific care settings were in- or excluded. All clinical trials and observational studies with a control group were included.

Studies were excluded if they were a validation study of a PROM, if the use of the PROM was to evaluate another intervention (e.g. treatment or follow-up strategy), when the study compared PROM intervention modalities (e.g. PROM A vs 
PROM B) or when the study focussed on children $(<$ 18 years).

\section{Risk of bias and methodological quality evaluation}

Risk of bias evaluation of all included studies was performed by two reviewers using the Cochrane Collaboration Risk of Bias Tool [25].

\section{Data synthesis and analysis}

Data extraction, synthesis and analysis were performed by two independent reviewers. The following study characteristics were extracted from each study: author, year, setting, study population, number of participants, intervention, control, method of data collection, education in interpretation yes/no, feedback received by patient or health care professionals yes/ no, patient outcomes and patient experiences. Feedback received by patients could be a summary of results or a treatment advice based on the results of the PROM.

Extracted outcomes and experiences were synthesized in a narrative matter and categorized into one of five categories: survival/mortality, symptoms/morbidity, health-related quality of life (HRQoL), patient satisfaction and process of care (number of discussed topics, duration consultation, emergency room visits, management/treatment actions, patient-doctor communication).

\section{Results}

\section{Study characteristics}

After removal of duplicates, 8341 references were identified through the initial search. An additional eight references were added by checking the reference list of previously published literature reviews $[18,19]$. Of 75 references eligible for fulltext screening, 22 met inclusion criteria and were included in the final analysis (Fig. 1).

Of the included studies, 19 (86\%) were randomized controlled trials and three (14\%) were sequential two-arm cohort studies. Most studies $(n=20,90 \%)$ were conducted in an outpatient clinic setting [16, 17, 26-43]. One study [44] was performed at a hospice and one study at an inpatient clinic [45]. Patients with various cancer types (including lung, breast, colorectal, gynaecologic, prostate, head and neck, lymphatic and prostate) and treatment modalities participated in the individual studies. The number of included patients ranged between 43 and 766 across the studies (Tables 1 and Appendix Table 6).

The majority of the studies were conducted in the USA $(n=8)$, followed by the UK $(n=5)$ and the Netherlands $(n=$ $3)$. Great diversity was seen in types of PROMs $(n=20)$, and

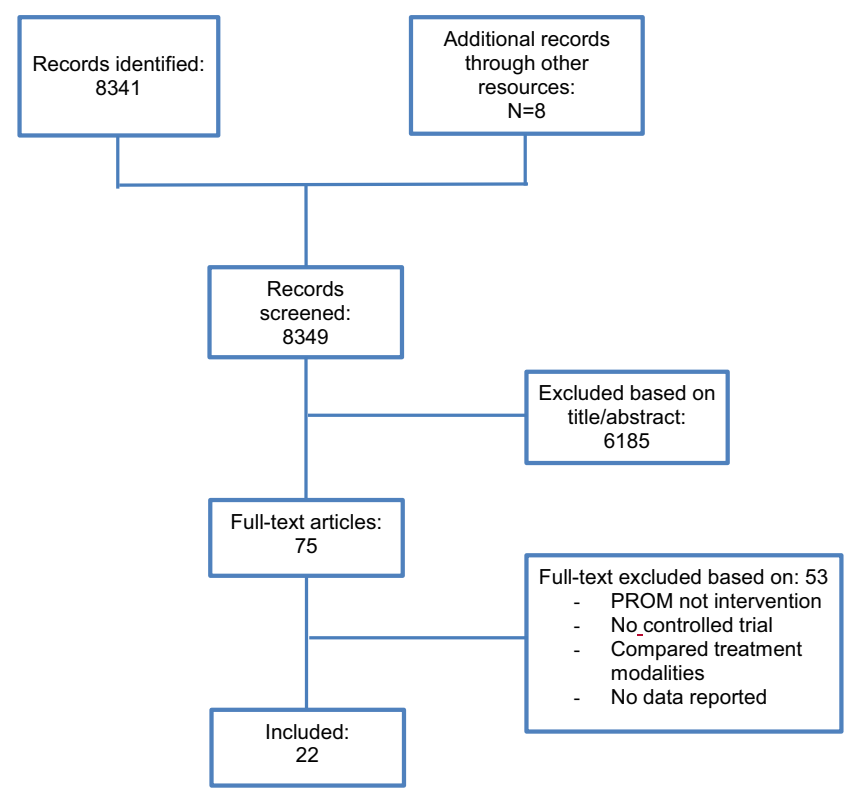

Fig. 1 Flow diagram of study identification and selection

several studies used more than one PROM (Table 2). The European Organisation for Research and Treatment of Cancer Quality of Life C30 (EORTC QLQ-C30) $(n=6)$, general symptoms on a numeric scale $(n=6)$ and the Hospital

Table 1 Characteristics of included studies

\begin{tabular}{lll}
\hline & $\begin{array}{l}\text { Number of } \\
\text { studies }\end{array}$ & $\begin{array}{l}\text { Portion of } \\
\text { total (\%) }\end{array}$ \\
\hline Setting & & \\
$\quad$ - Outpatient clinic & 20 & 90 \\
- In- and outpatient clinic & 1 & 5 \\
- Hospice & 1 & 5 \\
Study design & & \\
- Randomized controlled trial & 19 & 86 \\
- Cohort study & 3 & 14 \\
Types of cancer & & \\
- Various cancer types (including breast, & 16 & 72 \\
$\quad$ colorectal, lung, gynaecologic, head & & \\
$\quad$ and neck, etc.) & 4 & 18 \\
- Lung & 1 & 5 \\
- Lymphatic & 1 & 5 \\
- Prostate & & \\
Number of patients in included studies & 5 & 22 \\
- 1-100 & 10 & 45 \\
- 101-250 & 4 & 18 \\
- 251-500 & 3 & 14 \\
- > 500 & 7 & \\
Control group received & & \\
- No PROM (care as usual) & & \\
- PROM & & \\
\hline
\end{tabular}

${ }^{1}$ Percentages are rounded to the nearest percent 
Table 2 Characteristics of intervention

PROMs

- European Organisation for Research and Treatment of Cancer,

Quality of Life - Cancer 30 (EORTC QLQ-C30 )

- European Organisation for Research and Treatment of Cancer,

Quality of Life - Lung 30 (EORTC QLQ-LC13 )

- European Organisation for Research and Treatment of Cancer,

Quality of Life - Breast 23 (EORTC QLQ-BR23)

- European Organisation for Research and Treatment of Cancer,

Quality of Life - Colorectal 38 (EORTC QLQ-CR38 )

- General symptoms (on a numeric scale)

- Hospital Anxiety and Depression Scale (HADS)

- Symptom Tracking And Reporting (STAR)

- Supportive Care Needs Survey (SCNS)

- Functional Assessment of Cancer Therapy - General (FACT-G)

- Functional Assessment of Cancer Therapy - Prostate (FACT-P)

- MD Anderson Symptom Inventory (MDASI)

- Common Toxicity Criteria Adverse Events (CTCAE)

- Chemotherapy Symptom Assessment Scale (CSAS)

- Palliative Performance Scale (PPS)

- Memorial Symptom Assessment Scale (MSAS)

- Hospice Quality of Life (HQLI)

- Center for Epidemiological Studies-Depression (CES-D)

- Spiritual Needs Inventory (SNI)

- Short Portable Mental Status Questionnaire (SPMSQ)

- Therapy Related Symptom Checklist (TRSC)

Location of data collection

- Home

- Outpatient clinic

- In- and outpatient clinic

- Not reported

Methods of data collection

- Paper

- Electronic

- Paper and electronic

- Telephone

- Not reported

Feedback received by:

- Health care professional

- Patient and health care professional

- Not reported

Education in interpretation for health care professional

- Yes

- No

- Not reported

\begin{tabular}{|c|c|}
\hline 6 & 17 \\
\hline 3 & 9 \\
\hline 1 & 3 \\
\hline 1 & 3 \\
\hline 7 & 20 \\
\hline 3 & 9 \\
\hline 1 & 3 \\
\hline 1 & 3 \\
\hline 1 & 3 \\
\hline 1 & 3 \\
\hline 1 & 3 \\
\hline 1 & 3 \\
\hline 1 & 3 \\
\hline 1 & 3 \\
\hline 1 & 3 \\
\hline 1 & 3 \\
\hline 1 & 3 \\
\hline 1 & 3 \\
\hline 1 & 3 \\
\hline 7 & 32 \\
\hline 12 & 54 \\
\hline 1 & 5 \\
\hline 2 & 9 \\
\hline 6 & 27 \\
\hline 11 & 50 \\
\hline 1 & 5 \\
\hline 2 & 9 \\
\hline 2 & 9 \\
\hline 15 & 68 \\
\hline 6 & 27 \\
\hline 1 & 5 \\
\hline 6 & 27 \\
\hline 14 & 64 \\
\hline 2 & 9 \\
\hline
\end{tabular}

${ }^{1}$ Percentages are rounded to the nearest percent

Anxiety and Depression Scale (HADS) $(n=3)$ were the most commonly used PROMs in the included studies.

\section{Risk of bias}

Figure 2 summarizes the risk of bias of the included studies. Risk of bias (selection, performance, detection, attrition, reporting and other types of bias) was assessed using the Cochrane Risk of Bias Tool [25]. For the risk of bias assessment of each individual study, see Appendix Table 7. Random sequence generation risk of bias was as expected high in the three non-randomized controlled trials (RCTs) [30, 38, 42]. Allocation concealment was maintained in nine studies; in seven studies, this was not reported; and in five studies, there was a high risk of bias. All included studies were rated as high risk regarding performance bias as blinding of participants and personnel was not possible due to the nature of a PROM intervention. Twelve studies (57\%) were rated as low risk for detection bias. Ten studies (48\%) reported high rates of drop-out or loss to follow-up and were therefore rated as high risk of bias due to incomplete outcome data. Reporting bias was unclear in almost all studies. 
Fig. 2 Risk of bias presented as percentages across all included studies using Cochrane Risk of Bias Tool

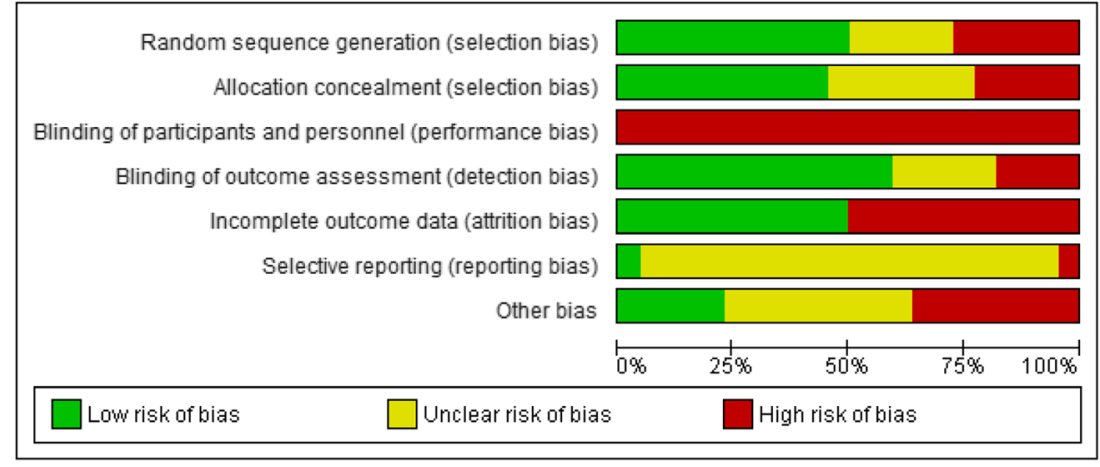

\section{PROM as intervention, with or without feedback to patients or health care professionals, compared with not using a PROM}

Of the 22 included studies, 15 studies were identified that compared the use of a PROM as the intervention to no PROM intervention [16, 26, 29-33, 36, 38, 43, 44]. Of these fifteen studies, in one study, the intervention was merely the use of a PROM [31]. In 14 studies, the use of the PROM was supplemented with feedback about the results $[16,26,29,30$, $32,33,36,38-44]$. In case feedback was provided, it was provided either to the HCP, the patient or both. In seven studies, the feedback was only available to the HCPs $[36,38-42$, 44]. In five studies, the feedback was provided to both patients and HCPs [16, 26, 29, 30, 32]. In two studies, the results were available for HCPs if patients proactively shared the feedback with them (Table 3) [33, 43].

\section{Patient outcomes—survival/mortality}

Only one study included survival as an outcome [26]. Basch et al. (2016) found that overall survival after 1 year was $76 \%$ in the PROM group versus 68\% in the non-PROM group $(p=$ $0.05)$. The study identified two important subgroups: computer-experienced and computer-inexperienced patients. Only one study included survival as an outcome [26]. Notably, in the subgroup of computer-inexperienced patients within the intervention group, the survival rate was significantly higher compared with the computer-inexperienced patients within the control group $(p=0.02)$. For the patients who were computer-experienced, no significant difference in survival was identified between the intervention and control group $(p=0.45)$. The authors suggested that computerinexperienced patients may have less-developed health communication skills and thereby benefit more from a structured program that incorporates self-reporting via PROMs.

\section{Patient outcomes-morbidity and symptoms}

Three studies evaluated symptoms as an outcome [31, 32, 44]. In the study of McMillan et al. (2011), patients with various cancer diagnoses who were admitted in a hospice filled out five PROMs that focused on symptoms, spiritual needs and HRQoL (i.e. PPS, MSAS, HQLI-4, CES-D, SNI, SPMSQ). The only outcome with a significant difference between the intervention group and control group was depression, as measured by the CES-D. While the depression scores declined significantly $(p=0.023)$ over time in both the intervention and control group, indicating an improvement in mental health, decline was significantly larger in the intervention group than in the control group $(p=0.027)$ [44].

In the study by Hoekstra et al. (2006), cancer patients receiving palliative treatment were randomized to either completing a symptom-based PROM (ten symptoms on a numeric 1-10 scale) every week at home or not completing a PROM. The symptoms evaluated were fatigue, pain, lack of appetite, shortness of breath, coughing, sleeplessness, nausea, constipation, diarrhoea and vomiting. It is unclear whether results were reported to the HCP and the patients. Significant differences were only identified for vomiting and constipation. The prevalence of these symptoms was lower in the intervention group [31].

Kearney et al. (2009) compared the use of a PROM (CTCAE and Chemotherapy Symptom Assessment Scale integrated into one questionnaire) for 14 days after a cycle of chemotherapy in breast, lung and colorectal cancer patients to care as usual without a PROM and found that the intervention group had a higher prevalence of hand-foot syndrome. Furthermore, the severity of hand-foot syndrome and associated levels of distress were also significantly higher. Other symptoms reported in the PROM did not differ significantly between the intervention and control group [32].

\section{Patient outcomes-HRQoL}

Studies performed by Basch et al. (2016), Velikova et al. (2004) and Williams et al. (2013) showed a positive effect on HRQoL when using a PROM compared with no PROM $[26,40,42]$. In these studies, patients in the intervention group reported a significant and clinically relevant improvement in HRQoL over time. Six studies did not identify significant difference between HRQoL scores between the intervention 


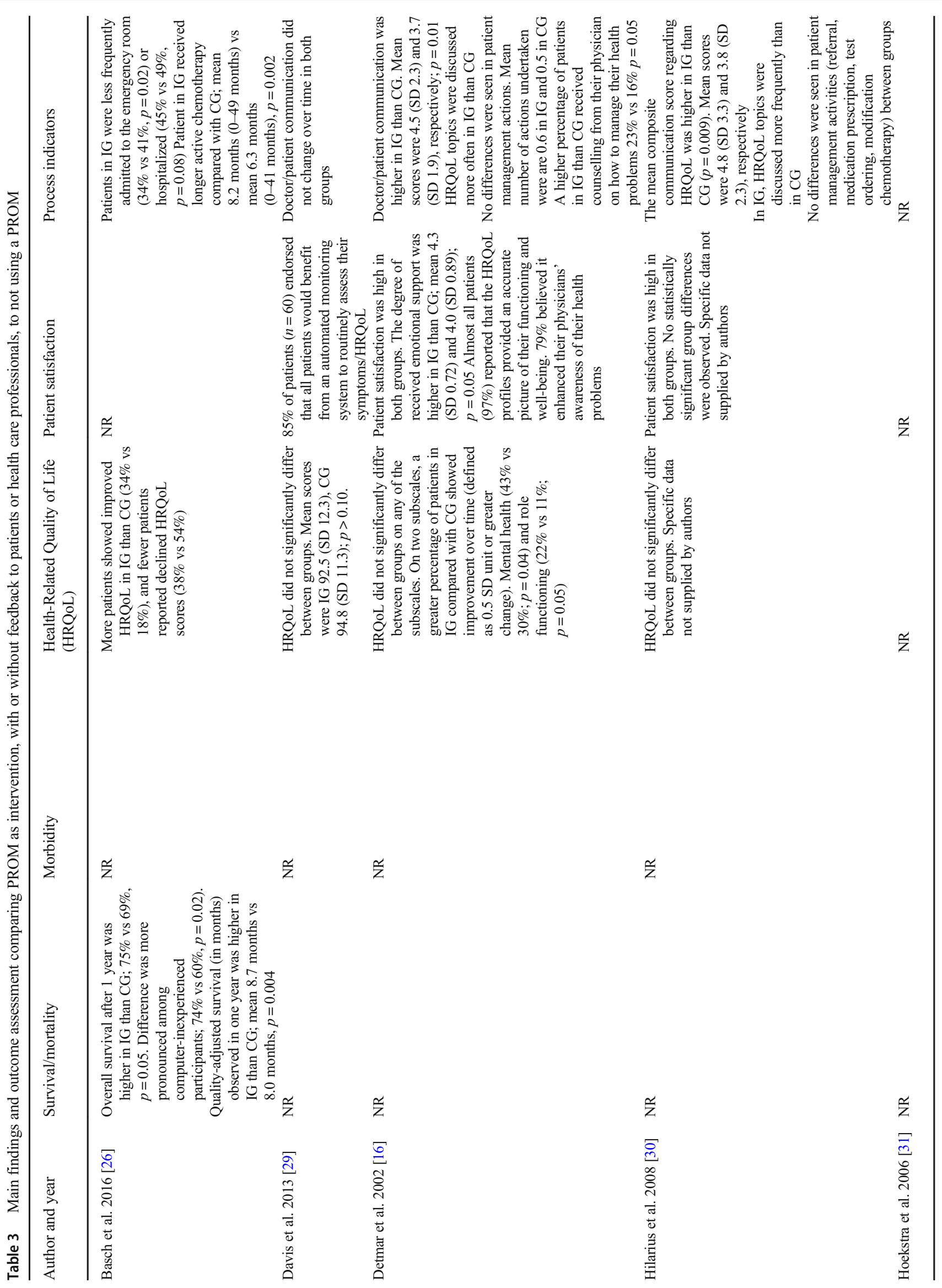




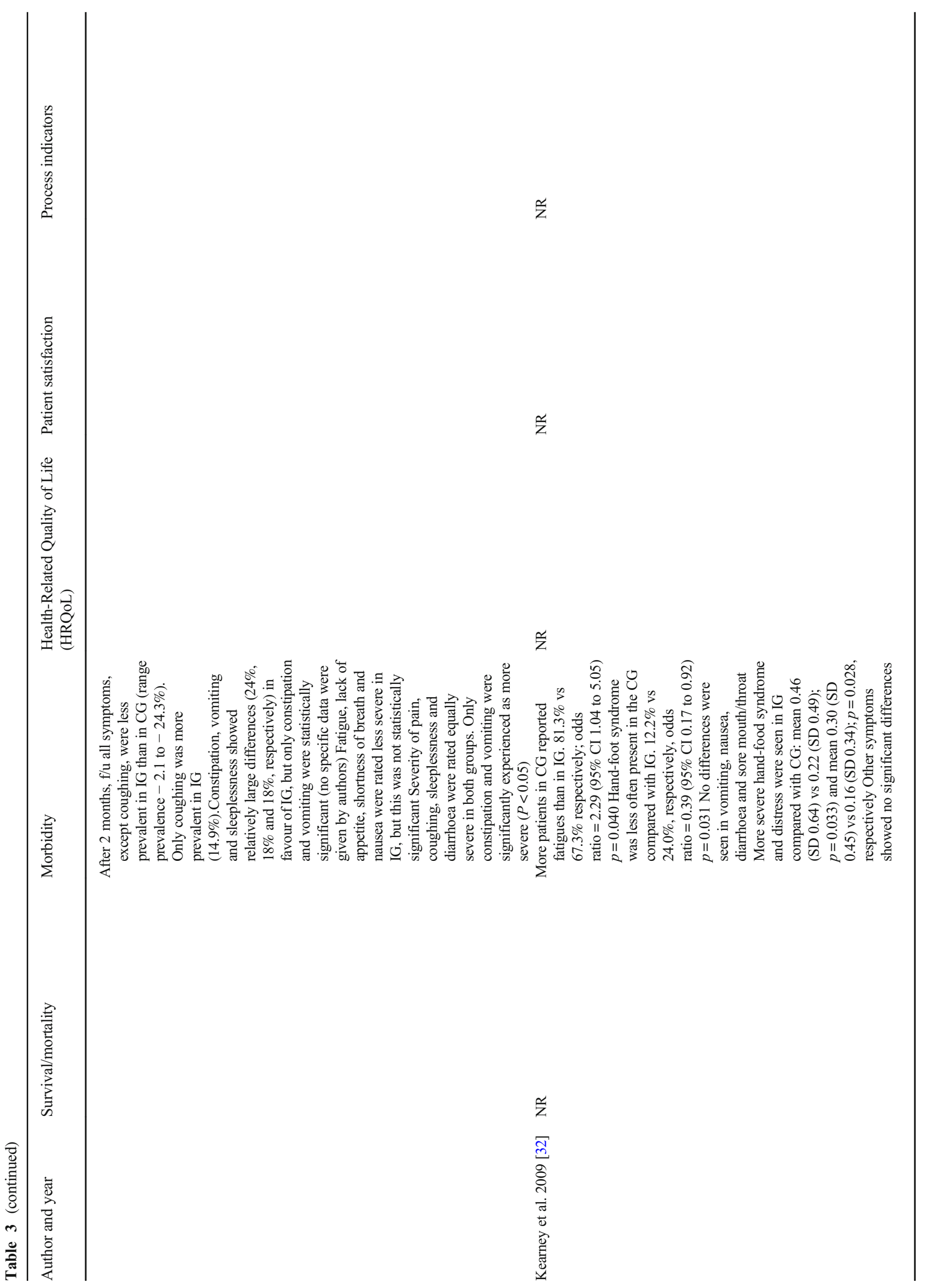




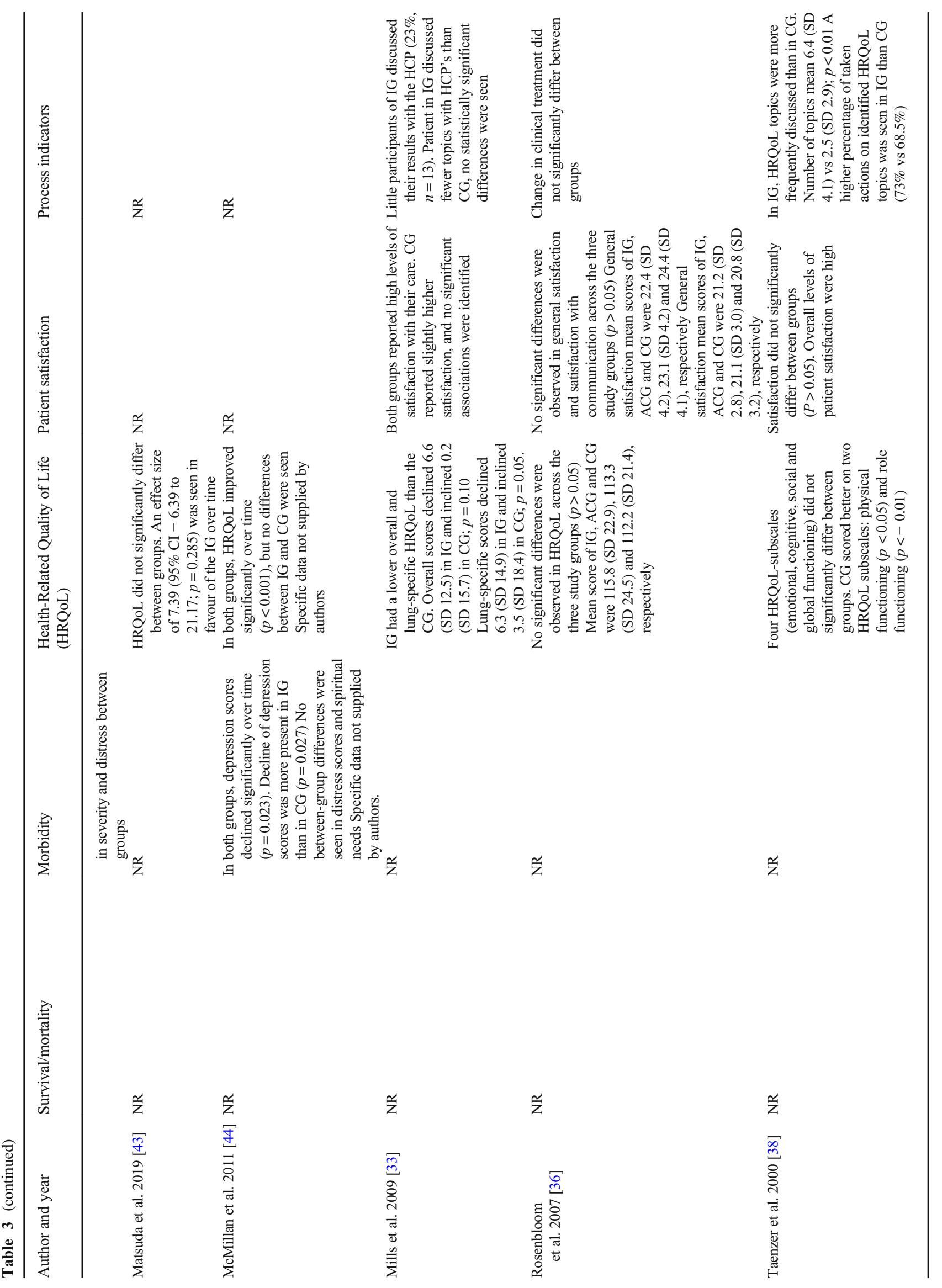




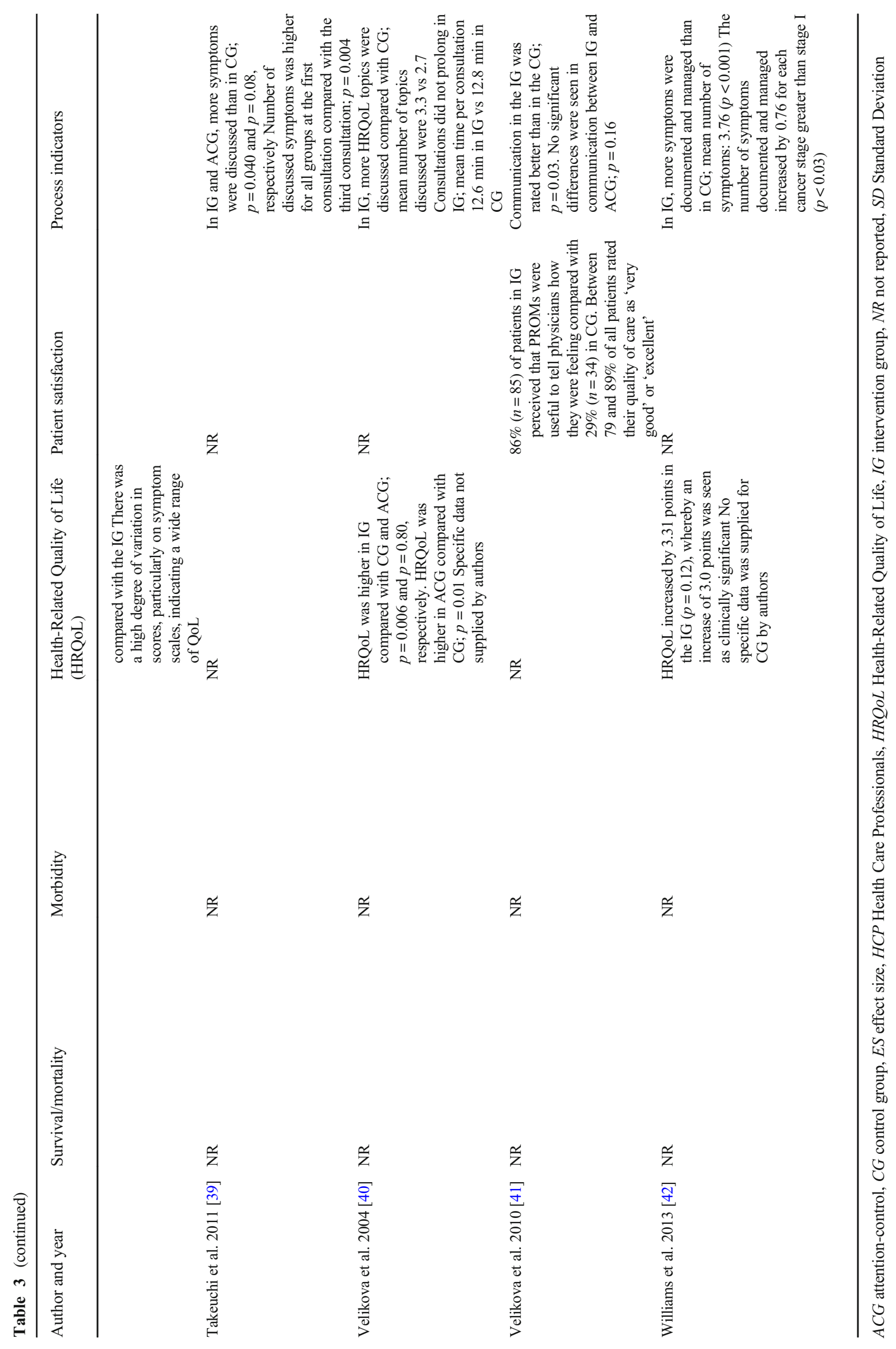


and control group [16, 29, 30, 36, 43, 44]. Mills et al. (2009) and Taenzer et al. (2000) found a negative effect when using a PROM [33, 38]. In the study of Mills et al. (2009), patients in the control group (i.e. no PROM) scored better on a lungspecific HRQoL scale, which included physical well-being, social/family well-being, emotional well-being, functional well-being and seven lung cancer-specific symptoms ( $p=$ 0.04) [33]. In the study of Taenzer et al. (2000), patients in the control group scored better on two specific HRQoL subdomains, physical functioning and role functioning $(p<0.05$ and $p<0.01$, respectively). In all other HRQoL domains, no significant differences were found between the PROM intervention and control group [38]. A lower reported HRQoL in the intervention group may be explained by the increased attention to HRQoL domains, resulting in more recognition and reporting of specific symptoms.

\section{Patient experiences—patient satisfaction}

In three studies, patients expressed the usefulness of a PROM [16, 29, 41]. They stated that PROMs were useful to tell their physician how they were feeling and that they represented an accurate representation of their functioning and well-being. The majority of patients, 79,85 and $86 \%$, respectively, were confident in that a PROM increased the awareness of their physician regarding their HRQoL and symptoms [16, 29, 41].

Four studies described no significant difference in patient satisfaction between patients completing a PROM and those who did not complete a PROM as part of their cancer care [30, $33,36,38]$.

\section{Process indicators}

The study performed by Basch et al. (2016) reported on emergency visits and hospital admissions. The intervention group (i.e. patients receiving a PROM with feedback) reported statistically significant fewer emergency visits and hospital admissions than the control group (i.e. no PROM)[26].

Evaluation of doctor-patient communication was described in four studies comparing a PROM intervention to no PROM $[16,29,30,41]$. In three studies, doctorpatient communication was rated better using a PROM [16, 30, 41], and one study [29] did not find any differences in the doctor-patient communication between the intervention and control group. In the study performed by Hilarius et al. (2008), more HRQoL topics were discussed in the intervention group than in the control group, but this did not lead to differences in patient management activities (e.g. referral, medication prescription, test ordering and modification chemotherapy) between both groups [30].
PROM as intervention with feedback to patients or health care professionals, compared with a control group in which PROMs were used without giving feedback to patients or health care professionals about the results

Seven studies were identified that compared use of a PROM with feedback to patients or health care professionals to use of a PROM without feedback to patients or health care professionals [17, 27, 28, 34, 35, 37, 45] (Table 4).

\section{Patient outcomes-morbidity and symptoms}

Five studies evaluated symptoms as an outcome [17, 28, 34, 37, 45]. In the study performed by Cleeland et al. (2011), an email was forwarded to the health care professionals in case a pre-set threshold of an alarming symptom (e.g. pain, distress, disturbed sleep, shortness of breath and constipation) was exceeded. Approximately $12 \%$ fewer emails regarding alarming symptoms were forwarded in the intervention group compared with the control group [28]. Ruland et al. (2010) found that in 75 leukaemia and lymphoma cancer patients who received feedback after completing a PROM, more symptoms had decreased compared with patients who had not received feedback about the findings of the PROM (ten of 19 symptoms vs two of 19 symptoms decreased). Of these ten symptoms, discomfort, eating/drinking, sleep/rest and sexuality were statistically significant in favour of the intervention group [45]. A favourable effect of using a PROM with feedback (compared with no feedback) was also seen in the study conducted by Strasser et al. (2016). The symptom distress score (including nine different symptoms rated on a 1-10 Likert scale) was significantly lower for the intervention than the control group over time $(p=0.003)$ [37]. In the study by Mooney et al. (2014), patients with various cancer types treated with chemotherapy were randomized to either reporting presence and severity of chemotherapy-related symptoms (rated on a 1-10 Likert scale) using an automated phone system with feedback to their physician or solely reporting chemotherapy-related symptoms using the identical automated phone system but without any feedback. No significant differences in symptom severity and distress scores were seen between the intervention and control group [34].

\section{Patient outcomes-HRQoL}

Only one article assessed HRQoL when comparing a PROM with or without feedback. Strasser et al. (2016) found a small, albeit significant, higher HRQoL in the group receiving a PROM with feedback. However, the difference between intervention group and control group was not considered clinically meaningful [37]. 


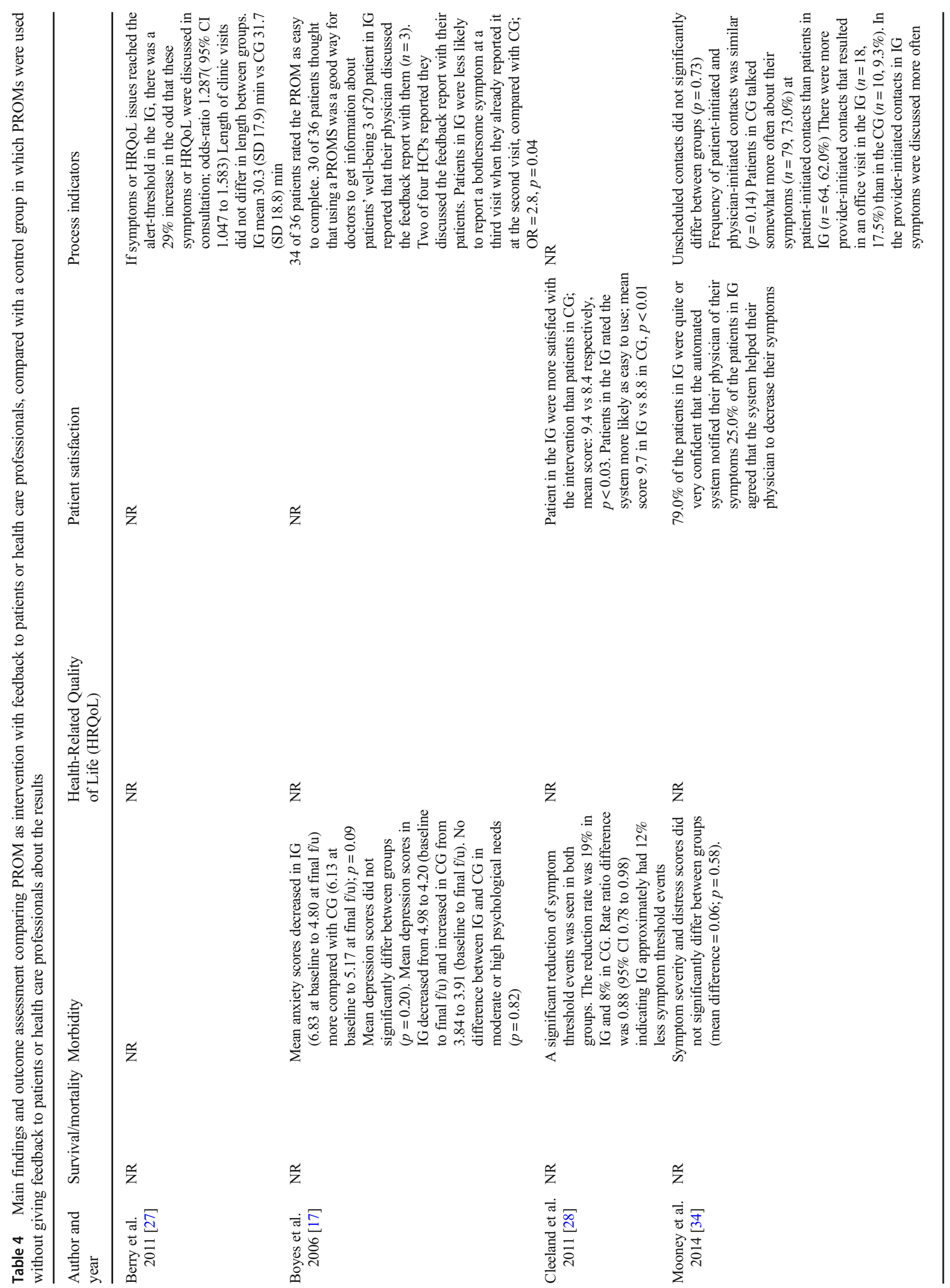




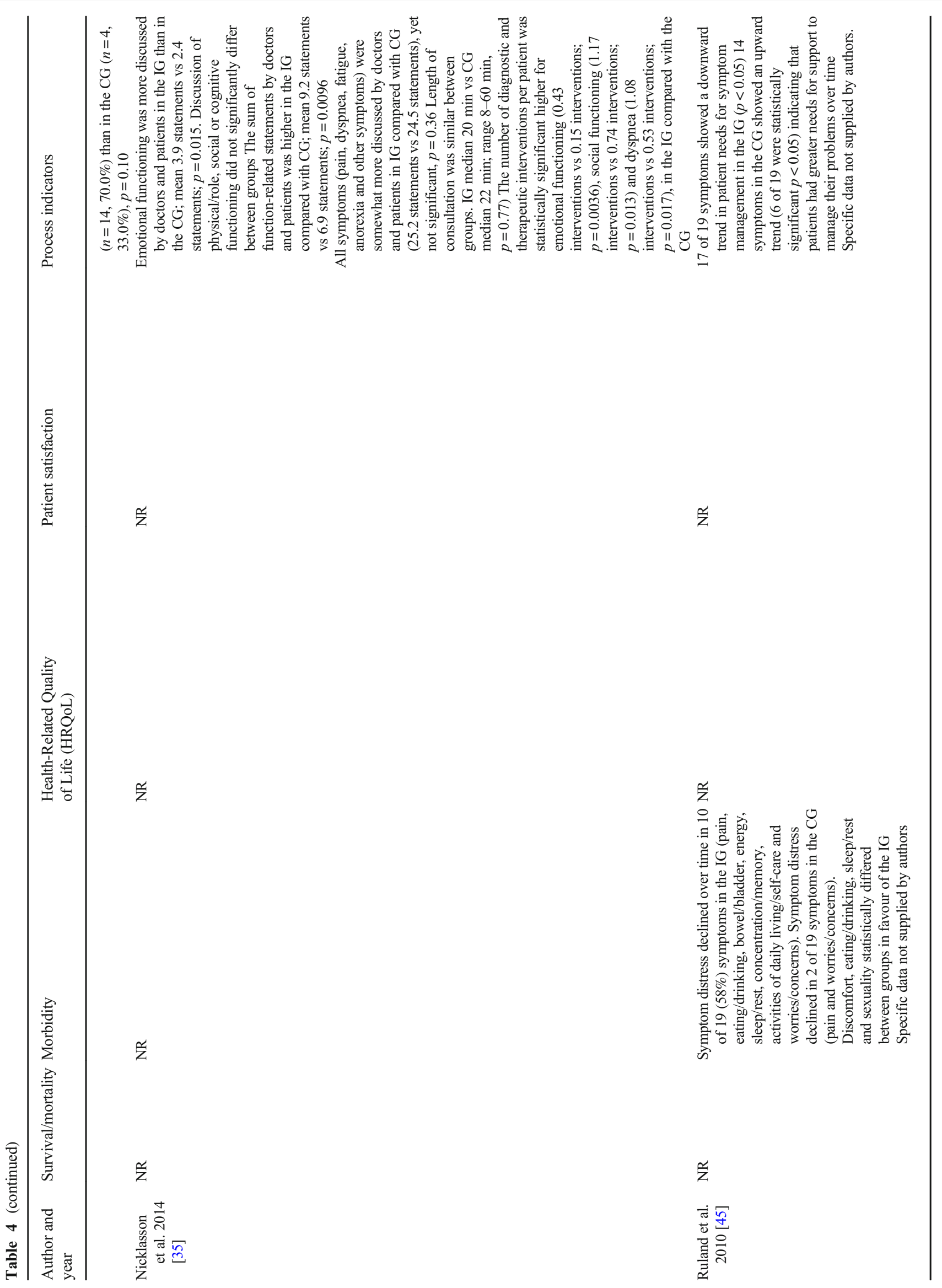


Two studies focused on the comparison of PROMs with feedback and without feedback reported on patient satisfaction $[28,34]$. In the study of Cleeland et al. (2011), higher levels of patient satisfaction were seen in the intervention group, compared with patients in the control group $(p<0.03)$. Mooney et al. (2014) found that $79 \%$ of patients were confident that the information they reported in the PROM would be noticed by their physician.

\section{Process indicators}

Mooney et al. (2014) reported that when health care professionals initiated the contact, more topics were discussed compared with when patients initiated contact [34]. In the study by Ruland et al. (2010), patients in the intervention group (i.e. PROM with feedback) and the control group (i.e. PROM without feedback) were asked to rate nineteen symptoms on a 1-10 Likert scale. In the intervention group, patients needed less symptom management in seventeen of nineteen symptoms. In six of these seventeen symptoms, significantly less symptom management or treatment was seen. In contrast to the intervention group, patients in the control group needed more symptom management in fourteen of nineteen symptoms, indicating that patients had greater needs for support in managing their symptoms. Significantly more symptom management was seen in six of these fourteen symptoms and all involved psychological needs - energy, sleep/rest, sexuality, mood/feelings, maintaining control over my situation and relationships [45]. In the study by Mooney et al. (2014), health care professionals treated both patients in the intervention group (i.e. receiving results of a PROM) and in the control group (i.e. not receiving results of a PROM). They found that the majority of health care professionals were satisfied with the PROM system and receiving alert reports, while $15 \%$ were not satisfied and did not read any of the received alert reports [34]. Berry et al. (2011) and Nicklasson et al. (2014) found no differences in consultation length between the group that received feedback on PROM results and the group that did not. Consultation time was not prolonged when health care professionals received feedback about PROM results.

\section{Discussion}

Primarily, we found positive or insignificant results after the use of a PROM in daily cancer care. Only few studies found negative effects of using a PROM. There appears to be an association between using a PROM in daily cancer care and better outcomes in specific symptoms, HRQoL, patient satisfaction and patient-physician communication. 
The first aim of this systematic review was to assess the effectiveness of PROMs and their effects on patient outcomes, patient experiences and process indicators. We identified 15 studies that compared the use of PROMs to not using PROMs. In twelve of the fifteen included studies, PROMs have shown a positive or no effect on survival, symptoms/morbidity, experienced HRQoL and patient satisfaction. Two studies reported a diminished experienced HRQoL.

HRQoL was the most commonly assessed outcome in studies evaluating PROM interventions. Eleven studies comparing a PROM versus no PROM used HRQoL as a primary or secondary outcome. While most studies $(9 / 11)$ found that a PROM intervention led to better HRQoL scores or unchanged HRQoL scores, there were two studies that found that a PROM intervention resulted in reduced HRQoL scores. Raising awareness regarding specific HRQoL domains (e.g. physical well-being, social/family well-being, emotional wellbeing, functional well-being and role functioning) without sufficient feedback by health care professionals could lead to increased worrying and uncontrolled thought processes which in turn may result in a poorer HRQoL $[33,38,46]$.

This review identified five studies that focused on symptoms as the outcome of the PROM intervention. Several symptoms (e.g. fatigue, constipation, vomiting) appeared to improve when completing symptom-based PROMs in the treatment trajectory, while others remained unaltered. A positive effect is likely to be related to contextual factors such as whether feedback to HCPs was provided and whether treatment strategies were then adapted to these findings. Most prominent, and somewhat inconsistent, were the findings regarding hand-foot symptoms, which were significantly more severe in the intervention group than in the control group in the study by Kearney et al. (2009) [32]. It is known that handfoot symptoms are poorly assessed in routine cancer care [47]. It may be the case that participants in the study by Kearney et al. (2009) randomized to the intervention group were directed more to their hand-foot syndrome symptoms as part of the PROM intervention compared with the control group. It is expected that in the longer term, these symptoms would improve due to the attention paid to these symptoms.

In three studies, patient satisfaction was higher when using a PROM compared with no PROM, whereas all other studies showed no differences in patient satisfaction. Patient satisfaction scores are known to be prone to possible ceiling effects and may have limited responsiveness due to high levels of satisfaction before the intervention, leaving little room for improvement [48]. It may therefore be more desirable to approach this specific outcome in a qualitative manner. An evaluation of the experiences and satisfaction using, for example, individual interviews can give more insight into the actual improvements in this outcome due to the PROM intervention.

Some studies found that doctor-patient communication was rated higher by patients when a PROM was used [16, 30, 41].
This seems plausible since the PROM intervention resulted in more HRQoL topics being addressed during the consultation than in usual care. Basch et al. (2016) reported fewer visits to the emergency room or admissions in the hospital. A formal costutility was not performed by the authors, yet for future use and to promote uptake and implementation, it would be interesting to assess whether PROM interventions are cost-effective [26].

In the second aim of the review, we identified the effect of providing feedback to patients and/or health care professionals on the outcomes reported in PROMs and narrowed down the first aim by focusing only on providing feedback. Seven studies described the effect of giving feedback. Similar to the findings of Kotronoulas et al. (2014) and Ishaque et al. (2019), this review found that receiving feedback on the completed PROMs resulted in better symptom control (i.e. less symptom threshold events, diminished symptom distress scores and decreased depression and anxiety scores), less need for symptom management, higher patient satisfaction and improved patientphysician communication compared with control groups not receiving any feedback $[17-19,27,28,35,45]$. It is likely that patient-doctor communication improved because the PROM intervention identifies more HRQoL topics relevant to the patient that are subsequently discussed than in the usual care setting, for example, problems with sleeping or cognitive functioning. Creating awareness regarding experienced symptoms and HRQoL among both patients and health care professionals seems to be essential in retaining better patient outcomes and experiences. Providing feedback to patients by health care professionals can be helpful in increasing this awareness.

A known barrier of PROM implementation is time constraint experienced by health care professionals [37, 49]. However, this review did not identify any differences in consultation length between using feedback of a PROM and not using feedback [27, 35].

Precautions must be taken in interpreting the results of the individual studies and the evidence synthesis since many studies were at high risk of bias and had insufficient power to detect significant differences in the outcomes assessed. The majority of the studies focussed only on statistical significant differences ( $p$ values) and did not mention whether this difference was also clinical relevant and meaningful. Only Basch et al. (2016), Detmar et al. (2002), Mills et al. (2009), Velikova et al. (2004) and Williams et al. (2013) reported on both statistical and clinical significance.

Compared with the previous review by Kotronolous et al. (2014), who showed that using PROMs increased the discussion of patient outcomes during consultations and that PROMs were associated with an improved symptom control and patient satisfaction, five new studies were included. This was less than we had anticipated from the noticeable increase in PROM popularity. A possible explanation could be that PROMs are often used as an instrument to assess outcomes of treatment modalities, but not as an intervention in itself. 
Moreover, the focus of this review was narrower than the review by Kotronoulas et al. in the sense that we did not include personalized management plans or clinical interventions linked to the PROM intervention that could have influenced our outcomes of interest (e.g. survival, symptoms/morbidity, HRQoL, patient satisfaction and process indicators).

We have synthesized the data retrieved from the reviewed studies in a narrative manner, due to the fact that a large variety was seen in types of cancer patients, treatment, types of PROMs, application of PROMs, evaluated outcomes and whether feedback was given to patients and caregivers. All studies differed in follow-up time, applied PROM(s), intervention assessment and study population (Table 4). It was therefore impossible to perform any type of quantitative synthesis or compare individual studies to each other. Categorization of assessed outcomes was chosen in order to be able to analyse the results. With this heterogeneity in mind, no recommendations can be made to which PROM intervention is most effective, what follow-up duration would be optimal and what method of data collection should be used. The majority of the studies included a variety of people with cancer as the study population and did not analysed their data stratified by type of cancer or treatment. Hence, a 'best practice' for a cancer type or treatment modality cannot be extracted from this review. Nevertheless, the review has identified important insight into the current available evidence regarding PROMs and their role in daily cancer care.

Further research should focus on the evaluation of the interventions (i.e. PROMs) that had an effect on the various outcomes, with a focus on the content of the intervention, and the impact of the contextual environment in which the PROM is implemented, health care professionals' attitudes and readiness to change and various implementation strategies on actual clinical outcomes.

\section{Conclusion}

In general, predominantly positive or insignificant findings were found in the use of a PROM in daily cancer care. There appears to be a trend towards better outcomes in specific symptoms, HRQoL, patient satisfaction and patientphysician communication. More positive effect were seen when feedback is provided to patient and/or health care professional, and it is thus highly recommended that this is always done. This review provides evidence that the use of PROMs, especially when combined with feedback to patient and/or health care professional, can improve outcomes and experiences on an individual patient level.
Acknowledgement The authors thank Lucas van Maaren for his valuable contribution to this work.

Funding information This study was funded by the Stichting Kwaliteitsgelden Medisch Specialisten (Quality Medical Specialists Foundation).

\section{Compliance with ethical standards}

\section{Conflict of interest}

- Caitlin Graupner, Merel L. Kimman and Stéphanie Breukink received research grants from the Stichting Kwaliteitsgelden Medisch Specialisten.

- All other authors declare that they have no conflict of interest.

- Authors have full control of all primary data and allow the journal to review the data.

\section{Appendix A}

Table 5 Search strategy as conducted in Ovid Medline and EMBASE

Search terms used

1. neoplasms/

2. (neoplasm* or cancer* or carcinoma* or oncology* or malignan* or tumo? $\mathrm{r}^{*}$ or leuk?emia* or sarcoma* or lymphoma* or blastoma* or melanoma*).ti,ab,kw.

3. patient-reported outcome measure/

4. (outcome* adj2 (measure* or tool* or assess* or score* or scale* or experience* or instrument* or questionnaire* or survey* or inventor*)).ti,ab,kw.

5. (Patient adj3 (outcome* or measure* or tool* or assess* or score* or scale* or satisfaction or experience* or instrument* or questionnaire* or survey* or inventor*)).ti,ab,kw.

6. ((self report* or self assess* or self monitor*) adj2 (outcome* or measure* or tool* or assess* or score* or scale* or satisfaction or experience* or instrument* or questionnaire* or survey* or inventor*)).ti,ab,kw.

7. PROM.ti,ab,kw.

8. PROMs.ti,ab,kw.

9. (PREM or PREMs).ti,ab,kw.

10. ((daily or routine* or consistent* or frequen* or regular* or standard or systematic) adj4 (use* or application or administ* or practice* or measure* or collection or assess* or utili?ation or monitor*)).ti,ab,kw.

11. 1 or 2

12. 3 or 4 or 5 or 6 or 7 or 8 or 9

13. 11 and 12

14. 10 and 11 and 12

$a b$ abstract, $a d j$ adjacency, $k w$ keyword, ti title 


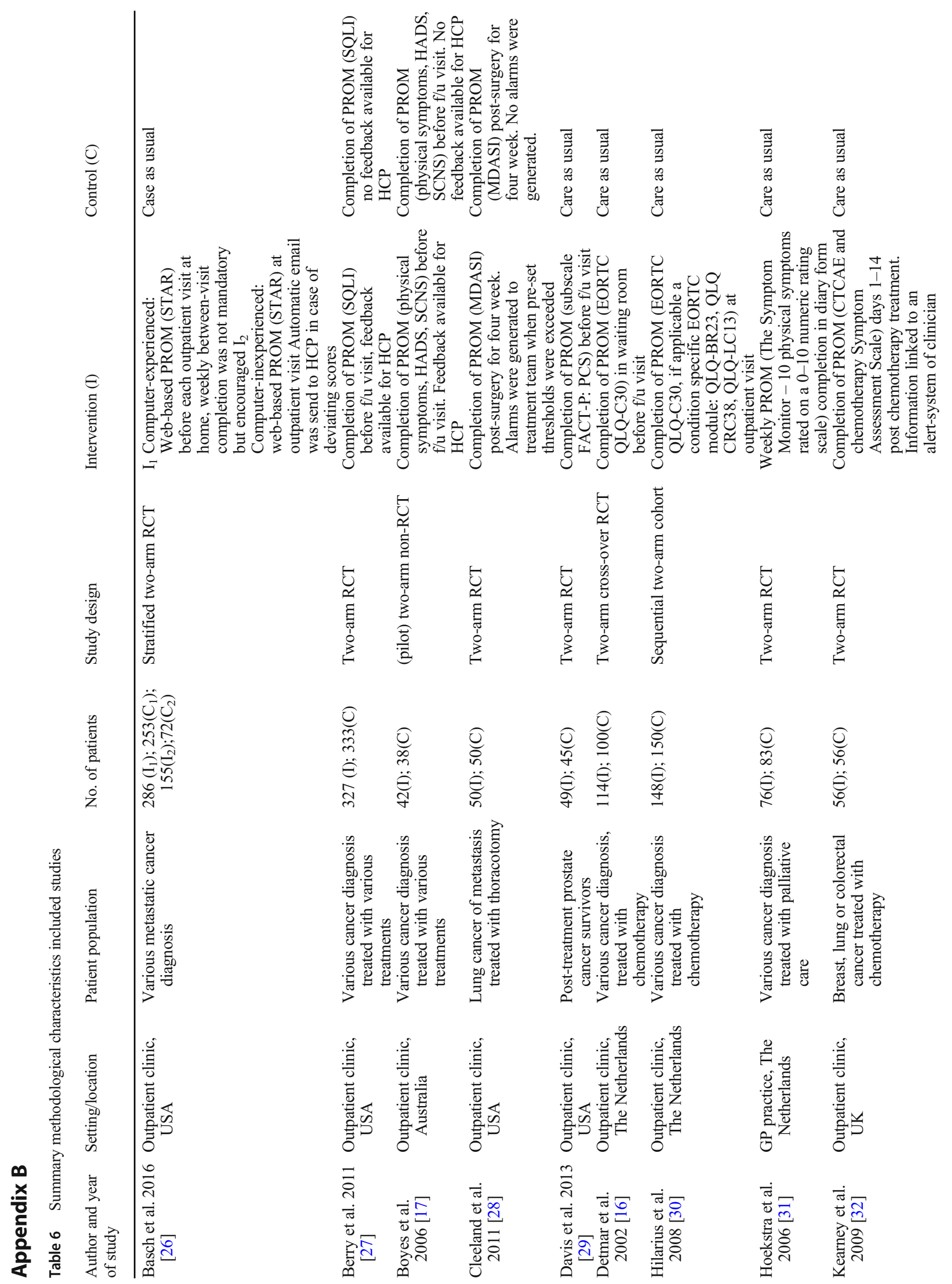




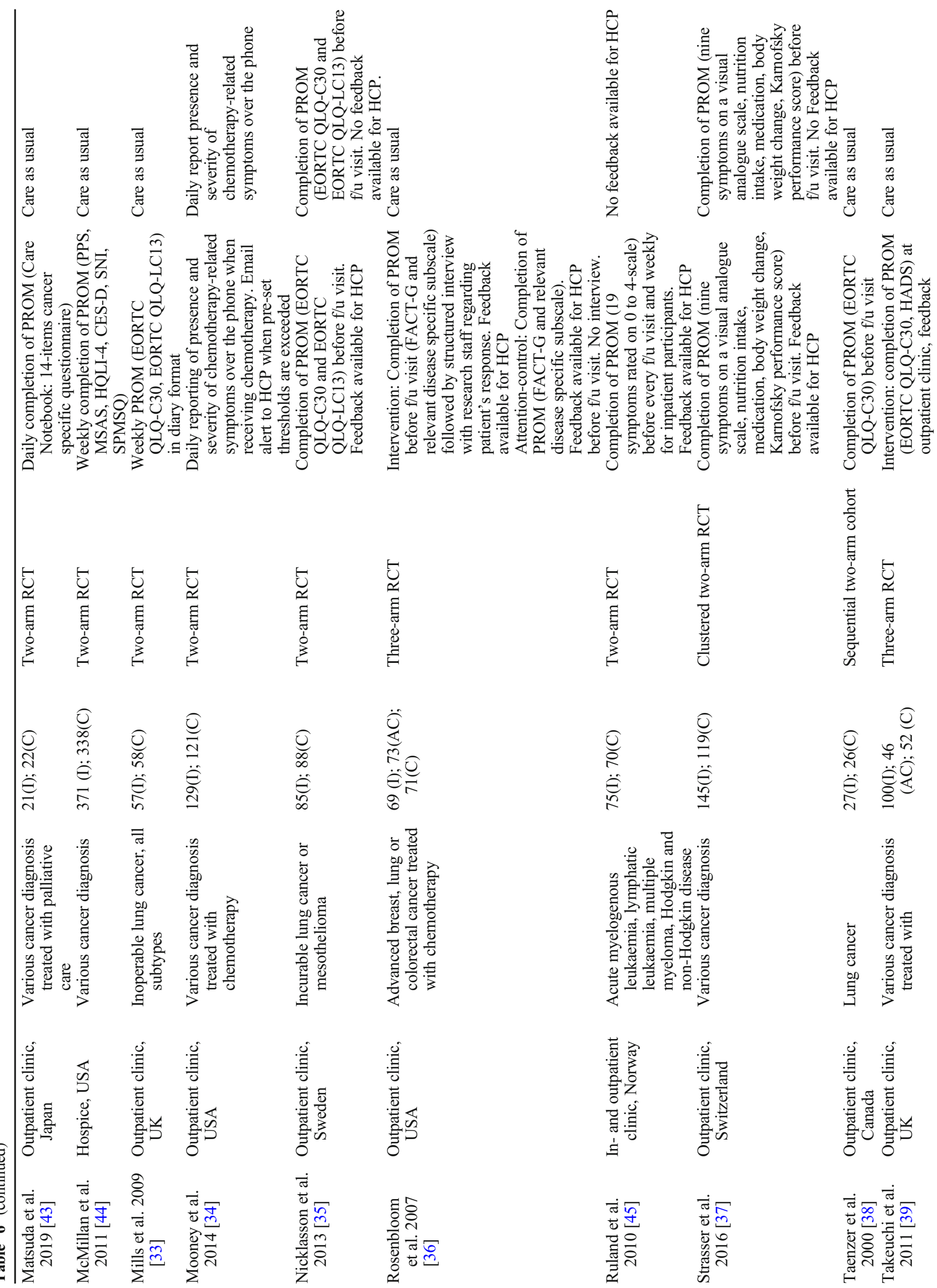




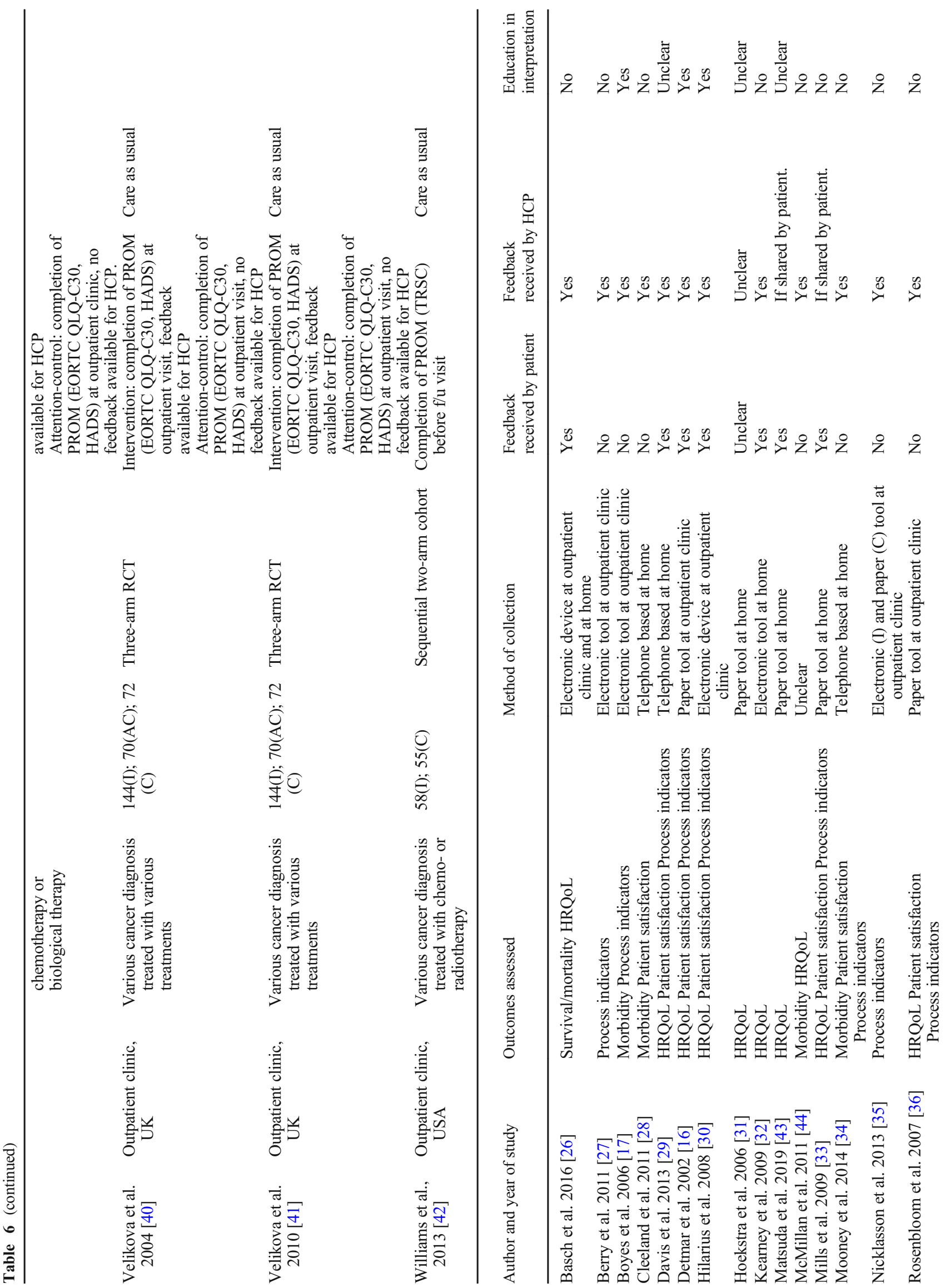




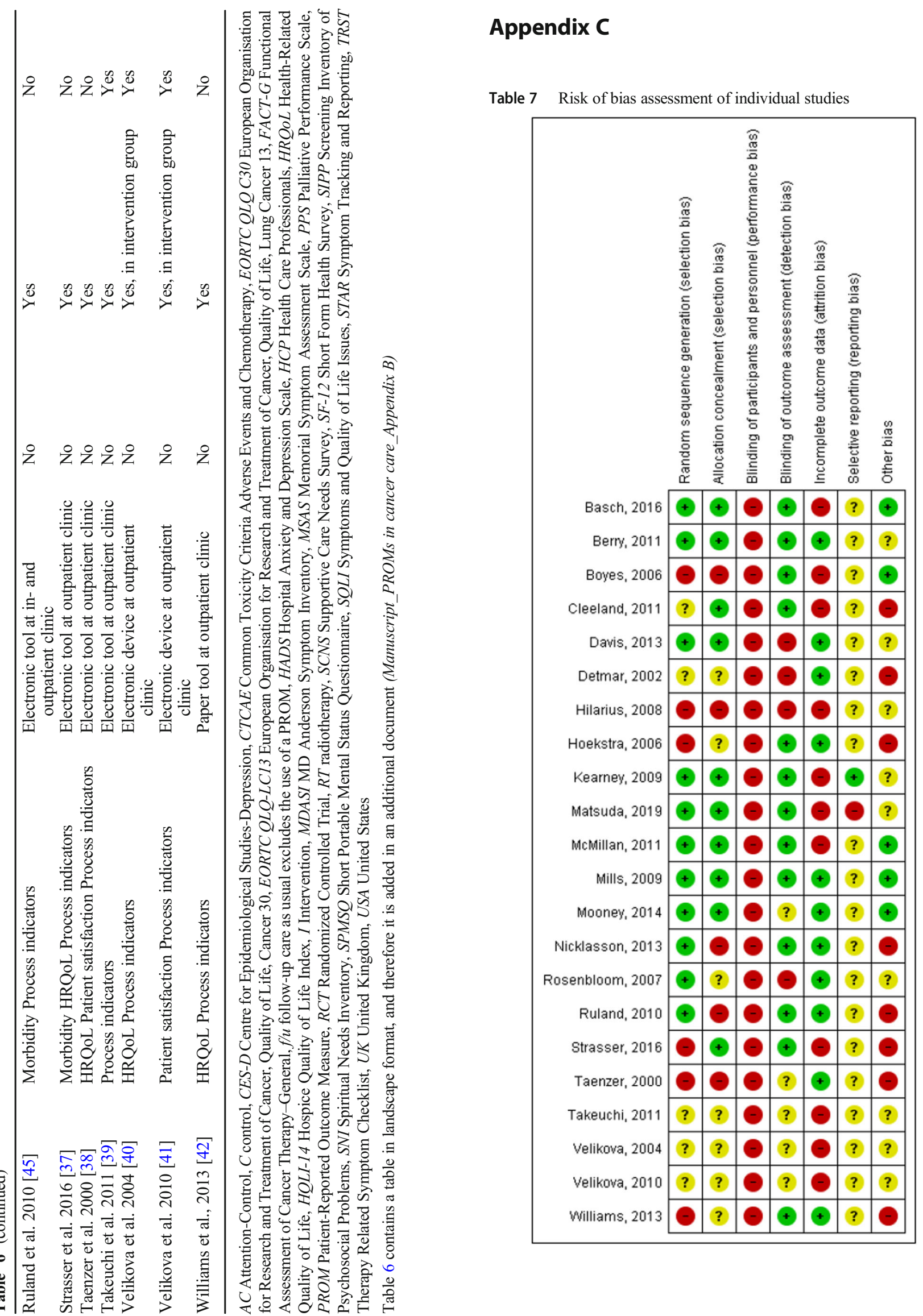


Open Access This article is licensed under a Creative Commons Attribution 4.0 International License, which permits use, sharing, adaptation, distribution and reproduction in any medium or format, as long as you give appropriate credit to the original author(s) and the source, provide a link to the Creative Commons licence, and indicate if changes were made. The images or other third party material in this article are included in the article's Creative Commons licence, unless indicated otherwise in a credit line to the material. If material is not included in the article's Creative Commons licence and your intended use is not permitted by statutory regulation or exceeds the permitted use, you will need to obtain permission directly from the copyright holder. To view a copy of this licence, visit http://creativecommons.org/licenses/by/4.0/.

\section{References}

1. Jemal A, et al (2017) Annual report to the nation on the status of cancer, 1975-2014, Featuring Survival. J Natl Cancer Inst 109(9)

2. Meadows KA (2011) Patient-reported outcome measures: an overview. Br J Commun Nurs 16(3):146-151

3. Fiteni $\mathrm{F}$ et al (2019) Clinical relevance of routine monitoring of patient-reported outcomes versus clinician-reported outcomes in oncology. In Vivo 33(1):17-21

4. Mak KS et al (2016) Defining a standard set of patient-centred outcomes for lung cancer. Eur Respir J 48(3):852-860

5. Ong WL et al (2017) A standard set of value-based patient-centered outcomes for breast cancer: the International Consortium for Health Outcomes Measurement (ICHOM) Initiative. JAMA Oncol 3(5): 677-685

6. Zerillo JA et al (2017) An international collaborative standardizing a comprehensive patient-centered outcomes measurement set for colorectal cancer. JAMA Oncol 3(5):686-694

7. Reilly CM et al (2013) A literature synthesis of symptom prevalence and severity in persons receiving active cancer treatment. Support Care Cancer 21(6):1525-1550

8. Henry DH et al (2008) Symptoms and treatment burden associated with cancer treatment: results from a cross-sectional national survey in the U.S. Support Care Cancer 16(7):791-801

9. Smith AW et al (2008) Cancer, comorbidities, and health-related quality of life of older adults. Health Care Financ Rev 29(4):41-56

10. Trautmann F et al (2016) Electronic real-time assessment of patientreported outcomes in routine care-first findings and experiences from the implementation in a comprehensive cancer center. Support Care Cancer 24(7):3047-3056

11. Howell D et al (2015) Patient-reported outcomes in routine cancer clinical practice: a scoping review of use, impact on health outcomes, and implementation factors. Ann Oncol 26(9):1846-1858

12. Chen J, Ou L, Hollis SJ (2013) A systematic review of the impact of routine collection of patient reported outcome measures on patients, providers and health organisations in an oncologic setting. BMC Health Serv Res 13:211

13. Higginson IJ, Carr AJ (2001) Measuring quality of life: using quality of life measures in the clinical setting. BMJ 322(7297):12971300

14. Nelson EC et al (2015) Patient reported outcome measures in practice. Bmj 350:g7818

15. Lipscomb J et al (2007) Patient-reported outcomes assessment in cancer trials: taking stock, moving forward. J Clin Oncol 25(32): $5133-5140$

16. Detmar SB et al (2002) Health-related quality-of-life assessments and patient-physician communication: a randomized controlled trial. JAMA 288(23):3027-3034
17. Boyes A et al (2006) Does routine assessment and real-time feedback improve cancer patients' psychosocial well-being? Eur J Cancer Care (Engl) 15(2):163-171

18. Kotronoulas $\mathrm{G}$ et al (2014) What is the value of the routine use of patient-reported outcome measures toward improvement of patient outcomes, processes of care, and health service outcomes in cancer care? A systematic review of controlled trials. J Clin Oncol 32(14): $1480-1501$

19. Ishaque $\mathrm{S}$ et al (2019) A systematic review of randomised controlled trials evaluating the use of patient-reported outcome measures (PROMs). Qual Life Res 28(3):567-592

20. van Egdom LSE et al (2019) Implementing patient-reported outcome measures in clinical breast cancer care: a systematic review. Value Health 22(10):1197-1226

21. Clohesy N, Schneiders A (2018) A preliminary investigation examining patient reported outcome measures for low back pain and utilisation amongst chiropractors in Australia: facilitators and barriers to clinical implementation. Chiropr Man Therap 26:38

22. Kaur M et al (2019) Implementing electronic patient-reported outcome measures in outpatient cosmetic surgery clinics: an exploratory qualitative study. Aesthet Surg J 39(6):687-695

23. Foster A et al (2018) The facilitators and barriers to implementing patient reported outcome measures in organisations delivering health related services: a systematic review of reviews. J Patient Rep Outcomes 2:46

24. Moher D et al (2009) Preferred reporting items for systematic reviews and meta-analyses: the PRISMA statement. Ann Intern Med 151(4):264-269 w64

25. Higgins JP et al (2011) The Cochrane Collaboration's tool for assessing risk of bias in randomised trials. BMJ 343:d5928

26. Basch E et al (2016) Symptom monitoring with patient-reported outcomes during routine cancer treatment: a randomized controlled trial. [Erratum appears in J Clin Oncol. 2016 Jun 20;34(18):2198; PMID: 27281229]. J Clin Oncol 34(6):557-565

27. Berry DL et al (2015) Exposure to a patient-centered, Web-based intervention for managing cancer symptom and quality of life issues: impact on symptom distress. J Med Internet Res 17(6):e136

28. Cleeland CS et al (2011) Automated symptom alerts reduce postoperative symptom severity after cancer surgery: a randomized controlled clinical trial. J Clin Oncol 29(8):994-1000

29. Davis $\mathrm{KM}$ et al (2013) Monitoring of health-related quality of life and symptoms in prostate cancer survivors: a randomized trial. J Support Oncol 11(4):174-182

30. Hilarius DL et al (2008) Use of health-related quality-of-life assessments in daily clinical oncology nursing practice: a community hospital-based intervention study. Cancer 113(3):628-637

31. Hoekstra J et al (2006) Using the symptom monitor in a randomized controlled trial: the effect on symptom prevalence and severity. J Pain Symptom Manag 31(1):22-30

32. Kearney N et al (2009) Evaluation of a mobile phone-based, advanced symptom management system (ASyMS) in the management of chemotherapy-related toxicity. Support Care Cancer 17(4):437-444

33. Mills ME et al (2009) Does a patient-held quality-of-life diary benefit patients with inoperable lung cancer? J Clin Oncol 27(1):70-77

34. Mooney KH et al (2014) Automated monitoring of symptoms during ambulatory chemotherapy and oncology providers' use of the information: a randomized controlled clinical trial. Support Care Cancer 22(9):2343-2350

35. Nicklasson M et al (2013) The impact of individual quality of life assessment on psychosocial attention in patients with chest malignancies: a randomized study. Support Care Cancer 21(1):87-95

36. Rosenbloom SK et al (2007) Assessment is not enough: a randomized controlled trial of the effects of HRQL assessment on quality of life and satisfaction in oncology clinical practice. Psycho-Oncology 16(12):1069-1079 
37. Strasser F et al (2016) The effect of real-time electronic monitoring of patient-reported symptoms and clinical syndromes in outpatient workflow of medical oncologists: E-MOSAIC, a multicenter cluster-randomized phase III study (SAKK 95/06). Ann Oncol 27(2):324-332

38. Taenzer P et al (2000) Impact of computerized quality of life screening on physician behaviour and patient satisfaction in lung cancer outpatients. Psychooncology 9(3):203-213

39. Takeuchi EE et al (2011) Impact of patient-reported outcomes in oncology: a longitudinal analysis of patient-physician communication. J Clin Oncol 29(21):2910-2917

40. Velikova $\mathrm{G}$ et al (2004) Measuring quality of life in routine oncology practice improves communication and patient well-being: a randomized controlled trial. J Clin Oncol 22(4):714-724

41. Velikova G et al (2010) Patients report improvements in continuity of care when quality of life assessments are used routinely in oncology practice: secondary outcomes of a randomised controlled trial. Eur J Cancer 46(13):2381-2388

42. Williams PD et al (2013) Therapy-related symptom checklist use during treatments at a cancer center. Cancer Nurs 36(3):245-254

43. Matsuda A et al (2019) Effectiveness of a self-monitoring quality of life intervention for patients with cancer receiving palliative care: a randomized controlled clinical trial. Asian Pac J Cancer Prev: APJCP 20(9):2795-2802
44. McMillan SC, Small BJ, Haley WE (2011) Improving hospice outcomes through systematic assessment: a clinical trial. Cancer Nurs 34(2):89-97

45. Ruland CM et al (2010) Effects of a computer-supported interactive tailored patient assessment tool on patient care, symptom distress, and patients' need for symptom management support: a randomized clinical trial. J Am Med Inform Assoc 17(4):403-410

46. Segerstrom SC et al (2003) A multidimensional structure for repetitive thought: what's on your mind, and how, and how much? J Pers Soc Psychol 85(5):909-921

47. Webster-Gandy JD, How C, Harrold K (2007) Palmar-plantar erythrodysesthesia (PPE): a literature review with commentary on experience in a cancer centre. Eur J Oncol Nurs 11(3):238-246

48. McHorney CA, Tarlov AR (1995) Individual-patient monitoring in clinical practice: are available health status surveys adequate? Qual Life Res 4(4):293-307

49. Snyder CF et al (2010) Relevant content for a patient-reported outcomes questionnaire for use in oncology clinical practice: putting doctors and patients on the same page. Qual Life Res 19(7):10451055

Publisher's note Springer Nature remains neutral with regard to jurisdictional claims in published maps and institutional affiliations. 